\title{
THE RANK CONJECTURE FOR NUMBER FIELDS
}

\author{
Armand Borel AND Jun YANG
}

\section{Introduction}

The rank conjecture states that two filtrations (the $\gamma$-filtration and the rank filtration) of the $K_{n}$-group $K_{n}(k)$ of an infinite field $k$ are complementary (see $[11,4.1]$ or [8]). Let $E$ denote a field of characteristic zero. In the case of a number field, and in view of known results on the $\gamma$ filtration, the rank conjecture is implied by two assertions pertaining to the homomorphism

$$
j_{i, m, n}: H_{i}\left(\mathbf{G L}_{m}(k) ; E\right) \longrightarrow H_{i}\left(\mathbf{G L}_{n}(k) ; E\right) \quad(m \leq n)
$$

induced by the standard inclusion

$$
j_{m, n}: \mathbf{G L}_{m}(k) \rightarrow \mathbf{G L}_{n}(k) \quad(m \leq n),
$$

namely,

$$
\begin{aligned}
& \operatorname{Im} j_{2 m-1, m, n} \supset P H_{2 m-1}\left(\mathbf{S L}_{n}(k) ; E\right), \\
& \operatorname{Im} j_{2 m-1, m-1, n} \cap P H_{2 m-1}\left(\mathbf{G L}_{n}(k) ; E\right)=\{0\} .
\end{aligned}
$$

for $n \gg m \geq 2$, where $P H_{i}$ is the space of primitive elements (1.4). The goal of [11] is to prove (R1) and (R2), but the argument is incomplete (see Section 6). More precisely, the proof of (R1) for quadratic fields in [11] is incorrect and the method there does not apply to $k=\mathbb{Q}$. Our first objective is to provide a complete proof (Section 5). To that effect, we first show (Theorem 2.1) that if $G$ is a simply connected, almost simple and isotropic $k$-group $G$, then $H^{\bullet}(G(k) ; \mathbb{R})$ is canonically isomorphic to the continuous (Eilenberg-MacLane) cohomology space $H_{\mathrm{ct}}^{\bullet}(G(k \otimes \mathbb{Q} \mathbb{R}) ; \mathbb{R})$. This, in turn, is an immediate consequence of the main theorem of [1] on the cohomology of $S$-arithmetic congruence subgroups of $G(k)$. The rank conjecture for number fields follows easily from this theorem, applied to $\mathbf{S L}_{n}$ viewed as a $k$-group and from some known facts about the continuous cohomology of $\mathbf{S L}_{n}(\mathbb{R})$ or $\mathbf{S L}_{n}(\mathbb{C})$ (Section 4).

Received September 19, 1994.

Most of the work on this note was carried out while the first named author enjoyed the hospitality of the Max-Planck-Institut für Mathematik in Bonn, Germany, as an A. v. Humboldt awardee. The second named author was supported in part by the NSF grant DMS-9401411. 
Finally, in Section 7 , we describe $H_{\bullet}\left(\mathbf{G L}_{n}(k) ; E\right)$ and show that $j_{i, m, n}$ is injective for all $i$ 's and surjective for $i \leq 2 m-1$.

\section{Notations and Review}

1.1. In this note, $k$ always stands for a number field, $V_{\infty}$ (resp. $V_{f}$ ) for the set of archimedean (resp. non-archimedean) places of $k$ and $V=V_{\infty} \cup V_{f}$. For $v \in V$, the completion of $k$ at $v$ is denoted by $k_{v}$. If $S \subset V$, then define $S_{f}=S \cap V_{f}$.

1.2. If $G$ is an affine $k$-group, and $S$ a finite subset of $V$, then $r(S, G)$ is the sum of the $k_{v}$-ranks of $G$ for $v \in S$. Moreover, as usual, we let

$$
G_{\infty}=G\left(k \otimes_{\mathbb{Q}} \mathbb{R}\right)=\prod_{v \in V_{\infty}} G\left(k_{v}\right),
$$

which is viewed as a real Lie group.

1.3. If $E$ is a commutative ring and $\Gamma$ a group, then $H^{i}(\Gamma ; E)$ and $H_{i}(\Gamma ; E)$ are the $i^{\text {th }}$ cohomology and homology group of $\Gamma$ with coefficients in the trivial $\Gamma$-module $E$, in the sense of Eilenberg-MacLane, and $H^{\bullet}(\Gamma ; E)$ (resp. $\left.H_{\bullet}(\Gamma ; E)\right)$ is the direct sum of the $H^{i}(\Gamma ; E)\left(\operatorname{resp} . H_{i}(\Gamma ; E)\right)$.

If $M$ is a real Lie group and $E$ is either $\mathbb{R}$ or $\mathbb{C}$, then $H_{\mathrm{ct}}^{i}(M ; E)$ denotes the $i^{\text {th }}$ continuous cohomology group of $M$ with respect to $E$, i.e., cohomology based on continuous (or differentiable) Eilenberg-MacLane cochains (see e.g. IX in [7]). Let $M^{\delta}$ be $M$ viewed as a discrete group (forget the topology). We let

$$
f: H_{\mathrm{ct}}^{\bullet}(M ; E) \longrightarrow H^{\bullet}\left(M^{\delta} ; E\right)
$$

be the natural map.

1.4. All fields in this paper are assumed to be commutative. Let $E$ be a field and

$$
A^{\bullet}=\bigoplus_{i=0}^{\infty} A^{i}
$$

be a graded algebra over $E$ which is connected, i.e., $A^{0}=E$. We let $I A=\sum_{i \geq 1} A^{i}$. Then $D A^{i}=A^{i} \cap(I A \cdot I A)$ is the space of decomposable elements in $A^{i}$ and $P A^{i}=A^{i} / D A^{i}$ the space of primitive elements in degree $i$. Let $A_{i}$ be the dual space to $A^{i}$ over $E$ and let

$$
A_{\bullet}=\bigoplus_{i=0}^{\infty} A_{i}
$$


Then, by definition, the space $P A_{i}$ of primitive elements in $A_{i}$ is the subspace on which $D A^{i}$ is zero. The duality between $A^{i}$ and $A_{i}$ defines one between $P A^{i}$ and $P A_{i}$.

\section{Theorem 2.1}

We first draw a consequence of the main result of [1].

Theorem 2.1. Let $G$ be a connected, simply connected, almost absolutely simple $k$-group of strictly positive $k$-rank $r_{k}(G)$. Then the natural homomorphism

$$
\mu: H_{c t}^{\bullet}\left(G_{\infty} ; \mathbb{R}\right) \longrightarrow H^{\bullet}(G(k) ; \mathbb{R})
$$

which is a composition of $f$ with the restriction map

$$
H^{\bullet}\left(G_{\infty}^{\delta} ; \mathbb{R}\right) \longrightarrow H^{\bullet}(G(k) ; \mathbb{R})
$$

is an isomorphism.

Proof. In this proof, $S$ is a finite subset of $V$ containing $V_{\infty}$ and at least one finite place. Let $\Gamma_{S}$ be a congruence $S$-arithmetic subgroup of $G(k)$. For example, if we fix an embedding $G \hookrightarrow \mathbf{G L}_{N}$ over $k$, we may take

$$
\Gamma_{S}=G\left(\mathcal{O}_{S}\right):=G(k) \cap \mathbf{G L}_{N}\left(\mathcal{O}_{S}\right),
$$

where $\mathcal{O}_{S}$ is the ring of $S$-integers of $k$. Then Theorem 1 in [1] asserts:

$$
H^{\bullet}\left(\Gamma_{S} ; \mathbb{R}\right)=H_{\text {ct }}^{\bullet}\left(G_{\infty} ; \mathbb{R}\right) \oplus H_{\text {cusp }}^{\bullet}\left(\Gamma_{S} ; \mathbb{R}\right) .
$$

The second summand on the right-hand side is the so-called cuspidal cohomology $([1],[6])$. The only property of $H_{\text {cusp }}^{\bullet}\left(\Gamma_{S} ; \mathbb{R}\right)$ relevant here is that it vanishes in degrees less than $r\left(S_{f} ; G\right)$. If $S$ runs through a strictly increasing sequence of subsets of $V$, then $r\left(S_{f} ; G\right) \rightarrow \infty$. Therefore, given $i \in \mathbb{N}$, we have

$$
H^{i}\left(\Gamma_{S} ; \mathbb{R}\right) \cong H_{\mathrm{ct}}^{i}\left(G_{\infty} ; \mathbb{R}\right)
$$

if $S$ is sufficiently large (e.g. if $\left|S_{f}\right|>i / r_{k}(G)$ ). The field $k$ is the inductive limit of the rings $\mathcal{O}_{S}(S \subset V)$, hence $G(k)$ is the inductive limit of the subgroups $G\left(\mathcal{O}_{S}\right)$. Therefore

$$
H_{\bullet}(G(k) ; \mathbb{R})=\varliminf_{S}^{\lim _{\bullet}} H_{\bullet}\left(G\left(\mathcal{O}_{S}\right) ; \mathbb{R}\right) .
$$

By duality, equation (3) implies that for a fixed $i$,

$$
H_{i}\left(G\left(\mathcal{O}_{S}\right) ; \mathbb{R}\right) \cong H_{i}(G(k) ; \mathbb{R})
$$


for sufficiently large $S$ (depending on $i$ ). Dualizing the statement again, we see that

$$
H^{i}(G(k) ; \mathbb{R}) \stackrel{\sim}{\rightarrow} H^{i}\left(G\left(\mathcal{O}_{S}\right) ; \mathbb{R}\right)
$$

for sufficiently large $S$, whence an isomorphism

$$
H^{i}(G(k) ; \mathbb{R}) \stackrel{\sim}{\rightarrow} H_{\mathrm{ct}}^{i}\left(G_{\infty} ; \mathbb{R}\right) .
$$

That it can be described as in the theorem can be seen by a discussion analogous to that in Section 4.8 of [11].

\section{Some Remarks}

3.1. For $G$ anisotropic over $k$, the isomorphism (2) and Theorem 2.1 are proved in [7, XIII, 3.5, 3.9], except that the formulation of $(2)$ is slightly different, since all the cohomology is cuspidal in that case. The argument used to deduce the theorem from (1) is the same as above.

3.2. The first named author proved in $[3]$ the existence of a constant $c(G)$ such that

$$
H_{\mathrm{ct}}^{i}\left(G_{\infty} ; \mathbb{R}\right) \longrightarrow H^{i}(\Gamma ; \mathbb{R})
$$

is an isomorphism for $i \leq c(G)$ and any arithmetic subgroup $\Gamma$ of $G(k)$. In [4], the result is extended to $S$-arithmetic subgroups and then to $G(k)$ with the same range. The main theorem of [1] shows that in fact, for $S$-arithmetic groups, the range increases with $S$, whence the improvement over the results of [4].

3.3. The proof of equation $2.1(2)$ consists of two main steps. First it is shown that $H^{\bullet}\left(\Gamma_{S} ; \mathbb{R}\right)$ is equal to the $L^{2}$-cohomology of $\Gamma_{S}$. This relies on [9], which is not yet published. The second and easier step consists in computing the $L^{2}$-cohomology. It is also carried out in [6].

3.4. The assumption that $G$ is simply connected is probably not essential. In fact, the second step in [6] does not need that assumption. However, the theorem does not extend to connected reductive groups. For instance, $H^{\bullet}\left(\mathbf{G L}_{1}(k) ; \mathbb{R}\right)$ is dual to an exterior algebra over an infinite dimensional vector space, while $H_{\mathrm{ct}}^{\bullet}\left(G_{\infty} ; \mathbb{R}\right)$ is finite dimensional.

\section{Application to $\mathbf{S L}_{m}(k)$}

In this section, we study the effect on homology and cohomology of the standard inclusion

$$
j_{m, n}: \mathbf{S L}_{m}(k) \longrightarrow \mathbf{S L}_{n}(k)
$$


4.1. Let $G_{n}=\mathbf{S L}_{n}$ be viewed as an algebraic group over $k$. Then

$$
H_{\mathrm{ct}}^{\bullet}\left(G_{n, \infty} ; \mathbb{R}\right) \cong \bigotimes_{v \in V_{\infty}} H_{\mathrm{ct}}^{\bullet}\left(\mathbf{S L}_{n}\left(k_{v}\right) ; \mathbb{R}\right)
$$

It is known that

$$
H_{\mathrm{ct}}^{\bullet}\left(\mathbf{S L}_{n}\left(k_{v}\right) ; \mathbb{R}\right)= \begin{cases}H^{\bullet}\left(\mathbf{S U}_{n} / \mathbf{S O}_{n} ; \mathbb{R}\right) & v \text { real }, \\ H^{\bullet}\left(\mathbf{S U}_{n} ; \mathbb{R}\right) & v \text { complex }\end{cases}
$$

The values of the right-hand side, computed in [2], are

$$
\begin{aligned}
H^{\bullet}\left(\mathbf{S U}_{n} / \mathbf{S O}_{n} ; \mathbb{R}\right) & = \begin{cases}\Lambda\left\langle x_{n, 5}, x_{n, 9}, \ldots, x_{n, 2 n-1}\right\rangle & n \text { odd } \\
\Lambda\left\langle x_{n, 5}, x_{n, 9}, \ldots, x_{n, 2 n-3}, e_{n}\right\rangle & n \text { even }\end{cases} \\
H^{\bullet}\left(\mathbf{S U}_{n} ; \mathbb{R}\right) & =\Lambda\left\langle x_{n, 3}, x_{n, 5}, \ldots, x_{n, 2 n-1}\right\rangle
\end{aligned}
$$

where $\Lambda\langle\rangle$ denotes a graded exterior algebra over the generators listed in \langle\rangle , the second subscript of $x_{n, j}$ denotes the degree and $e_{n}$ is of degree $n$.

4.2. One can easily study the effect of $j_{m, n}^{\bullet},(m<n)$ using (3), (4). For consistent choices of the generators, we have

$$
\begin{aligned}
& j_{m, n}^{i}\left(x_{n, i}\right)=x_{m, i} \quad(i \leq 2 m-1), \\
& j_{m, n}^{i}\left(x_{n, i}\right)=0 \quad(i>2 m-1), \\
& j_{m, n}^{n}\left(e_{n}\right)=0 \quad(n \text { even }) .
\end{aligned}
$$

Therefore

$$
j_{m, n}^{i}: P H_{\mathrm{ct}}^{i}\left(G_{n, \infty} ; \mathbb{R}\right) \longrightarrow P H_{\mathrm{ct}}^{i}\left(G_{m, \infty} ; \mathbb{R}\right)
$$

is an isomorphism if $i$ is odd and $\leq 2 m-1$.

As a corollary, we obtain the following

Theorem 4.3. For any field $E$ of characteristic 0,

(a) $j_{m, n}^{i}: P H^{i}\left(\boldsymbol{S} \boldsymbol{L}_{n}(k) ; E\right) \rightarrow P H^{i}\left(\boldsymbol{S} \boldsymbol{L}_{m}(k) ; E\right)$ and

(b) $j_{i, m, n}: P H_{i}\left(\boldsymbol{S L}_{m}(k) ; E\right) \rightarrow P H_{i}\left(\boldsymbol{S L}_{n}(k) ; E\right) \quad$ are isomorphisms for $i$ odd, $i \leq 2 m-1$.

(c) $j_{m-1, n}^{2 m-1}\left(P H^{2 m-1}\left(\boldsymbol{S} \boldsymbol{L}_{n}(k) ; E\right)\right)=\{0\}$.

The assertion (a) is a consequence of (8) and 2.1, the assertion (b) follows by duality and (c) from (2.1) and (6), with $m$ replaced by $m-1$. 


\section{The Rank Conjecture}

Theorem 5.1. The rank conjecture is true for all number fields.

As recalled in the introduction, we have to prove the two assertions (R1) and (R2) stated there.

By duality and 3.9 in [5], the inclusion $\mathbf{S L}_{n}(k) \rightarrow \mathbf{G L}_{n}(k)$ induces an isomorphism

$$
P H_{2 m-1}\left(\mathbf{S L}_{n}(k) ; E\right) \stackrel{\sim}{\rightarrow} P H_{2 m-1}\left(\mathbf{G L}_{n}(k) ; E\right), \quad(n \gg m \geq 2) .
$$

Therefore, it suffices to prove (R1) with GL replaced by SL. Then it follows from 4.3(a).

By duality and (1), (R2) is equivalent to

$$
j_{n, m}^{2 m-1}\left(P H^{2 m-1}\left(\mathbf{S L}_{n}(k) ; E\right)\right)=0 \quad(n \geq m \geq 2),
$$

which follows from 4.3(c).

REMARK: We have given a recent reference for (1) or the dual assertion, but it was known before. For instance, it can be derived from the homology stability of $\mathbf{G L}_{n}$ proved by Suslin [10] and the fact that (1) is true with $n$ replaced by $\infty$, since both sides are then naturally isomorphic to $K_{2 m-1}(k) \otimes E$ by the Milnor-Moore theorem.

\section{Erratum to [11]}

The main goal of [11] is to prove (R1), (R2) above for $k \neq \mathbb{Q}$. The proof of (R2) given there is valid for all $k$ 's, but it implicitly uses the fact that

$$
P H^{2 n-1}\left(\mathbf{G L}_{n}(k) ; \mathbb{R}\right) \cong P H^{2 n-1}\left(\mathbf{S L}_{n}(k) ; \mathbb{R}\right)
$$

discussed above. Also the proof there made use of Proposition 4.9, which is unnecessary. Once it is known that the map

$$
P H^{2 n-1}(\mathbf{G L}(k) ; \mathbb{R}) \rightarrow H^{2 n-1}(\mathbf{G L}(k) ; \mathbb{R}) \rightarrow H^{2 n-1}\left(\mathbf{G L}_{m}(k) ; \mathbb{R}\right)
$$

is trivial for $m<n$, the lower rank conjecture follows by duality.

The proof of (R1) for quadratic fields in [11] is not valid, however. First of all, the estimate for $\alpha(J)$ on p. 305 in the imaginary quadratic

field case is incorrect. Hence the injectivity assertion for $j^{2 n-1}$ on top of p. 305 is not proved for imaginary quadratic fields, and in fact is clearly false for $n=2$. Even when the injectivity on top of p. 305 holds, the argument in 4.12 in [11], based on 4.9, does not quite give (R1). There is some confusion on the notion of primitive elements. More precisely, a subspace $D H_{i}$ of $H_{i}\left(\mathbf{S L}_{n}(k) ; \mathbb{R}\right)$ is defined, which is a supplement to $P H_{i}\left(\mathbf{S L}_{n}(k) ; \mathbb{R}\right)$. The argument in $[11]$ shows that

$$
j_{2 m-1, m, n}\left(H_{2 m-1}\left(\mathbf{S L}_{m}(k) ; \mathbb{R}\right)\right)
$$


contains a supplement to $D H_{i}$, but not necessarily $P H_{2 m-1}\left(\mathbf{S L}_{n}(k) ; \mathbb{R}\right)$ itself.

\section{Homology of $\mathbf{G L}_{n}(k)$ In Characteristic Zero}

7.1. Given a field $F$ and $n \in \mathbb{N}$, we identify $x \in F^{*}$ to the diagonal matrix $d(x) \in G L_{n}(F)$ with all diagonal entries equal to 1 , except for the first one, which is equal to $x$. The group $\mathbf{G L}_{n}(k)$ is the semi-direct product of the subgroup

$$
Z=\left\{d(x) \mid x \in F^{*}\right\}
$$

and the normal subgroup $\mathbf{S L}_{n}(F)$. The group $Z$ operates by inner automorphisms on the cohomology (or homology) of $\mathbf{S L}_{n}(F)$ and we let $\delta(x)$ be the automorphism so defined. In case $F$ is a topological field, we have similarly an operation $\delta(x)$ on the continuous cohomology. We first determine the $Z$-invariants in $H^{\bullet}\left(\mathbf{S L}_{n}(F) ; \mathbb{R}\right)$, when $F=k, k_{v}\left(v \in V_{\infty}\right)$.

We refer to Section 4 (2), (3), (4) for the description of $H_{\mathrm{ct}}^{\bullet}\left(\mathbf{S L}_{n}\left(k_{v}\right) ; \mathbb{R}\right)$.

Lemma 7.2. We keep the previous notation. Then

$$
H_{c t}^{\bullet}\left(\boldsymbol{S \boldsymbol { L } _ { n }}\left(k_{v}\right) ; \mathbb{R}\right)^{k_{v}^{*}}= \begin{cases}H_{c t}^{\bullet}\left(\boldsymbol{S L}_{n}\left(k_{v}\right) ; \mathbb{R}\right) & (v \text { complex or } n \text { odd }) \\ \Lambda_{\mathbb{R}}\left\langle x_{n, 5}, x_{n, 9}, \ldots, x_{n, 2 n-3}\right\rangle & (v \text { real, } n \text { even }) .\end{cases}
$$

Proof. The spaces $\mathbf{S U}_{n} / \mathbf{S O}_{n}$ and $\mathbf{S U}_{n}$ are deformation retracts of $\mathbf{S L}_{n}(\mathbb{C}) / \mathbf{S L}_{n}(\mathbb{R})$ and $\mathbf{S L}_{n}(\mathbb{C})$ respectively. We can therefore replace $\mathbf{S U}_{n}$ and $\mathbf{S O}_{n}$ by $\mathbf{S L}_{n}(\mathbb{C})$ and $\mathbf{S} \mathbf{L}_{n}(\mathbb{R})$ in $(2),(3)$, (4) of Section 4 . Note that on the left-hand side of these relations we have the singular cohomology groups of some manifolds, so any connected group of homeomorphisms acts trivially on them. If $v$ is complex, then $k_{v}^{*}$ is connected, whence the lemma in that case. The same is true for the group $\mathbb{R}_{+}^{*}$ of strictly positive real numbers if $v$ is real so that, in that case, we are reduced to studying the effect of $\delta(-1)$. Since it belongs to $\mathbf{O}_{m}$, we may again use the compact picture.

The map

$$
p^{\bullet}: H^{\bullet}\left(\mathbf{S U}_{n} / \mathbf{S O}_{n} ; \mathbb{R}\right) \rightarrow H^{\bullet}\left(\mathbf{S U}_{n} ; \mathbb{R}\right)
$$

induced by the projection

$$
p: \mathbf{S U}_{n} \longrightarrow \mathbf{S U}_{n} / \mathbf{S O}_{n}
$$

is injective on odd dimensional generators (cf. [2]). Since $p^{\bullet}$ commutes with $\delta(-1)$, this implies that $\delta(-1)$ acts trivially on the subalgebra of $H^{\bullet}\left(\mathbf{S U}_{n} / \mathbf{S O}_{n} ; \mathbb{R}\right)$ generated by the $x_{n, i}$. For $n$ odd, this concludes the proof. 
If $n$ is even, there remains to be seen that

$$
\delta(-1)\left(e_{n}\right)=-e_{n} .
$$

The class $e_{n}$ is the image by transgression in the bundle

$$
\mathbf{S O}_{n} \rightarrow \mathbf{S U}_{n} \rightarrow \mathbf{S U}_{n} / \mathbf{S O}_{n}
$$

of a class $c_{n-1} \in H^{n-1}\left(\mathbf{S O}_{n} ; \mathbb{R}\right)$ and $\delta(-1)$ commutes with transgression. So we are reduced to showing that

$$
\delta(-1)\left(c_{n-1}\right)=-c_{n-1} .
$$

The class $c_{n-1}$ is the image of a generator $d_{n-1}$ of $H^{n-1}\left(\mathbf{S}^{n-1} ; \mathbb{R}\right)$ under the map

$$
q^{\bullet}: H^{\bullet}\left(\mathbf{S}^{n-1} ; \mathbb{R}\right) \longrightarrow H^{\bullet}\left(\mathbf{S O}_{n} ; \mathbb{R}\right)
$$

induced by the projection

$$
q: \mathbf{S O}_{n} \longrightarrow \mathbf{S}^{n-1} \text {. }
$$

The projection $q$ commutes with $\delta(-1)$ acting by inner automorphism on $\mathbf{S O}_{n}$ and via $d(-1)$ on $\mathbb{R}^{n}$. Since $d(-1)$ changes the orientation, our assertion follows.

7.3. For each $v \in V_{\infty}$, let $\sigma_{v}$ be an associated embedding. When $v \in V_{\mathbb{R}}$, $\sigma_{v}$ is unique. If $v$ is complex, then take $\sigma_{v}$ to be one of the two conjugate embeddings associated to $v$. The homomorphism $\mu$ of Section 2 (1) may be written as a tensor product

$$
\mu=\bigotimes_{v \in V_{\infty}} \mu_{v}
$$

where $\mu_{v}$ is the composition

$$
H_{\mathrm{ct}}^{\bullet}\left(\mathbf{S L}_{n}\left(k_{v}\right) ; \mathbb{R}\right) \rightarrow H^{\bullet}\left(\mathbf{S L}_{n}\left(k_{v}\right)^{\delta} ; \mathbb{R}\right) \stackrel{\sigma_{v}^{\bullet}}{\rightarrow} H^{\bullet}\left(\mathbf{S L}_{n}(k) ; \mathbb{R}\right) .
$$

Let

$$
\begin{aligned}
S_{n, v}^{\bullet} & =\mu_{v}\left(H_{\mathrm{ct}}^{\bullet}\left(\mathbf{S L}_{n}\left(k_{v}\right) ; \mathbb{R}\right)^{k_{v}^{*}}\right) \\
S_{n}^{\bullet} & =\bigotimes_{v \in V_{\infty}} S_{n, v}^{\bullet} .
\end{aligned}
$$

Then 2.1(1) may be written

$$
H^{\bullet}\left(\mathbf{S L}_{n}(k) ; \mathbb{R}\right) \cong S_{n}^{\bullet} \otimes \Lambda_{\mathbb{R}}\left\langle\mu\left(e_{n, 1}\right), \ldots, \mu\left(e_{n, r}\right)\right\rangle,
$$

where $e_{n, i}$ is the Euler class in the factor associated to $v_{i}$, and $V_{\mathbb{R}}=$ $\left\{v_{1}, \ldots, v_{r}\right\}$ is the set of real places, or equivalently real embeddings, of $k$. 
Lemma 7.4. We have the equalities

(a) $H^{\bullet}\left(\boldsymbol{S} \boldsymbol{L}_{n}(k) ; \mathbb{R}\right)^{k^{*}}=S_{n}^{\bullet}$,

(b) $S_{m}^{\bullet}=j_{m, n}^{\bullet}\left(H^{\bullet}\left(\boldsymbol{S} \boldsymbol{L}_{n}(k) ; \mathbb{R}\right)\right) \quad(n>m \geq 2)$.

Moreover,

(c) $j_{m, n}^{i}: S_{n}^{i} \rightarrow S_{m}^{i}$ is an isomorphism for $i \leq 2 m-1$.

Proof. We note first that

$$
\mu_{v} \circ \delta\left(\sigma_{v}(x)\right)=\delta(x) \circ \mu_{v} \quad\left(v \in V_{\infty} ; x \in k^{*}\right)
$$

on $H_{\mathrm{ct}}^{\bullet}\left(\mathbf{S L}_{n}\left(k_{v}\right) ; \mathbb{R}\right)$, as follows from the definition. Let

$$
N=\left\{x \in k^{*} \mid \sigma_{v}(x)>0, v \in V_{\mathbb{R}}\right\}, \quad C=k^{*} / N .
$$

By (2) and the initial remark in the proof of $7.2, N$ acts trivially on $H^{\bullet}\left(\mathbf{S L}_{n}(k) ; \mathbb{R}\right)$, hence

$$
H^{\bullet}\left(\mathbf{S L}_{n}(k) ; \mathbb{R}\right)^{k^{*}}=S_{n}^{\bullet} \otimes\left(\Lambda_{\mathbb{R}}\left\langle\mu\left(e_{n, 1}\right), \ldots, \mu\left(e_{n, r}\right)\right\rangle\right)^{C} .
$$

By weak approximation, $C=(\mathbb{Z} / 2 \mathbb{Z})^{r}$, i.e., given any pattern of signs $\left\{\epsilon_{\sigma_{v}} \mid v \in V_{\mathbb{R}}\right\}$, there exists $x \in k^{*}$ such that sign $\sigma_{v}(x)=\epsilon_{\sigma_{v}}$, for $v \in V_{\mathbb{R}}$. Then (2) and 7.2 show that the second factor on the right-hand side of (4) reduces to the constants, and (a) is proved. Now (b) and (c) follow from Section 4 (5), (6), (7).

REMARK: In view of 7.4(b), $S_{m}$ has a natural $\mathbb{Q}$-structure $S_{m, \mathbb{Q}}$, namely the image of the rational cohomology of $\mathbf{S L}_{n}(k)$. We let

$$
S_{m, E}=S_{m, \mathbb{Q}} \otimes_{\mathbb{Q}} E .
$$

Theorem 7.5. We have the isomorphisms

$$
H^{\bullet}\left(k^{*} ; H^{\bullet}\left(\boldsymbol{S L}_{n}(k) ; E\right)\right)=H^{\bullet}\left(k^{*} ; E\right) \otimes S_{n, E}^{\bullet}=H^{\bullet}\left(\boldsymbol{G} \boldsymbol{L}_{n}(k) ; E\right) .
$$

Proof. The algebra $H^{\bullet}\left(k^{*} ; H^{\bullet}\left(\mathbf{S L}_{n}(k) ; E\right)\right)$ is the abutment of a spectral sequence associated to the group extension (see (3))

$$
1 \rightarrow N \rightarrow k^{*} \rightarrow C \rightarrow 1
$$

in which, as usual,

$$
E_{2}^{p, q}=H^{p}\left(C ; H^{q}\left(N ; H^{\bullet}\left(\mathbf{S L}_{n}(k) ; E\right)\right)\right) .
$$

Since $N$ acts trivially on $H^{\bullet}\left(\mathbf{S L}_{n}(k) ; E\right)$, we have

$$
H^{q}\left(N ; H^{\bullet}\left(\mathbf{S L}_{n}(k) ; E\right)\right) \cong H^{q}(N ; E) \otimes H^{\bullet}\left(\mathbf{S L}_{n}(k) ; E\right) .
$$


The group $C$ is finite and $E$ is of characteristic zero, hence

$$
E_{2}^{p, q}= \begin{cases}\left(H^{q}(N ; E) \otimes H^{\bullet}\left(\mathbf{S L}_{n}(k) ; E\right)\right)^{C}, & p=0 \\ 0, & p \neq 0\end{cases}
$$

But $k^{*}$ is commutative, hence acts trivially on the cohomology of $N$ and we get

$$
E_{2}^{0, q}=H^{q}(N ; E) \otimes\left(H^{\bullet}\left(\mathbf{S L}_{n}(k) ; E\right)\right)^{C} .
$$

Therefore by 7.4 and the remark to 7.4 ,

$$
H^{\bullet}\left(k^{*} ; H^{\bullet}\left(\mathbf{S L}_{n}(k) ; E\right)\right)=H^{\bullet}(N ; E) \otimes S_{n, E} .
$$

The same computation shows that the spectral sequence of (6) for the trivial $k^{*}$-module $E$ collapses and yields

$$
H^{\bullet}(N ; E)=H^{\bullet}\left(k^{*} ; E\right),
$$

and the first equality follows from (11) and (12).

To prove the second equality, we consider the commutative diagram of extensions

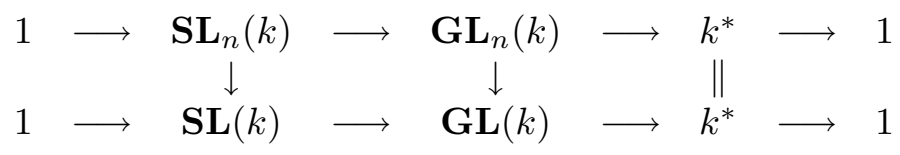

where as usual, $\mathbf{S L}(k)$ and $\mathbf{G L}(k)$ are defined to be the inductive limits of $\mathbf{S L}_{n}(k)$ and $\mathbf{G} \mathbf{L}_{n}(k)$ respectively, and the vertical arrows are natural inclusions. It is known that the spectral sequence of the second row of (13) is trivial, hence

$$
H^{\bullet}(\mathbf{G L}(k) ; E)=H^{\bullet}\left(k^{*} ; E\right) \otimes H^{\bullet}(\mathbf{S L}(k) ; E)
$$

(see 3.9 in [5]). Lemma 7.4(b) and stability imply that the image of

$$
j_{n, \infty}^{\bullet}: H^{\bullet}(\mathbf{S L}(k) ; E) \rightarrow H^{\bullet}\left(\mathbf{S L}_{n}(k) ; E\right)
$$

is $S_{n, E}$.

Let $\left\{E_{r}^{\prime}\right\}$ be the spectral sequence of the first row of (13). Then the previous remark and the first part of 7.5 show that

$$
j_{n, \infty}: E_{2}^{p, q} \rightarrow E_{2}^{\prime p, q}
$$

is surjective. Since the first spectral sequence collapses at $E_{2}$, the same is true for the second one, whence the second part of 7.5.

A priori, there might be an extension problem since we have proved this only for $E_{\infty}=E_{2}$. However, since the "fibre factor" $S_{n, E}$ is an 
exterior algebra on odd dimensional generators, hence free among graded anti-commutative algebras over $E$, this is not an issue.

Corollary 7.6. Let $m, n \in \mathbb{N}, m \leq n$. Then

$$
\text { (a) } \quad j_{m, n}^{i}: H^{i}\left(\boldsymbol{G} \boldsymbol{L}_{n}(k) ; E\right) \rightarrow H^{i}\left(\boldsymbol{G} \boldsymbol{L}_{m}(k) ; E\right)
$$

is surjective for all $i$ 's and injective for $i \leq 2 m-1$;

(b) $\quad j_{i, m, n}: H_{i}\left(\boldsymbol{G} \boldsymbol{L}_{m}(k) ; E\right) \rightarrow H_{i}\left(\boldsymbol{G L}_{n}(k) ; E\right)$

is injective for all $i$ 's and surjective for $i \leq 2 m-1$.

Proof. The first assertion follows from 7.4, 7.5, the structure of $S_{n, E}^{\bullet}$ given by 7.2 and 4.2 . The second one follows from the first by duality.

Remark 7.7. We see in particular that $j_{i, m, n}$ is an isomorphism for $i \leq$ $2 m-1$, which gives a sharper version of the "upper" rank conjecture. We recall that by 3.4 in [10], this is true for any infinite field and any trivial coefficients if $i \leq m$. We also understand that Suslin had conjectured the injectivity of $j_{i, m, n}$ for all $i$ 's for infinite fields.

\section{References}

1. D. Blasius, J. Franke, F. Grunewald, Cohomology of S-arithmetic groups in the number field case, Invent. Math. 116 (1994), 75-93.

2. A. Borel, Sur la cohomologie des espaces fibrés principaux et des espaces homogènes de groupes de Lie compacts, Ann. Math. 57 (1953), 115-207.

3. Stable real cohomology of arithmetic groups, Ann. Sci. Ecole Norm. Sup. (4) 7 (1974), 235-272.

4. Stable real cohomology of arithmetic groups II, Prog. Math. 14 (1981), 21-55, Birkhäuser-Boston.

5. _ Values of zeta-functions at integers, cohomology and polylogarithms, preprint.

6. A. Borel, J.-P. Labesse, J. Schwermer, On the construction of cuspidal cohomology of $S$-arithmetic subgroups of reductive groups, Preprint.

7. A. Borel, N. Wallach, Continuous cohomology, Discrete Subgroups, and Representations of Reductive Groups, Princeton University Press, Princeton, 1980.

8. J-L. Cathelineau, Homologie du groupe linéaire et polylogarithmes, Sém. Bourbaki, 1992-93, Exp. 772, Astérisque 216, 1993, 311-341, Soc. Math. France.

9. J. Franke, Harmonic analysis in weighted $L_{2}$-spaces, in preparation.

10. A. A. Suslin, Homology of $\boldsymbol{G L}_{n}$, characteristic classes and Milnor K-theory, LNM, 1046 (1984), 357-375, Springer-Verlag.

11. J. Yang, On the real cohomology of arithmetic groups and the rank conjecture for number fields. Ann. Scient. Éc. Norm. Sup. 25 (1992), 287-306.

Institute for Advanced Study, Princeton, NJ 08540

E-mail address: borel@math.ias.edu

Department of Mathematics, Duke University, Durham, NC 27708-0320

E-mail address: yang@math.duke.edu 